\title{
Educational Simulator for Frequency Estimation using ANN
}

\author{
Nedim Aktan Yalçın and *Fahri Vatansever \\ Faculty of Engineering, Electrical-Electronics Eng. Dept., Bursa Uludağ University, Turkey
}

\begin{abstract}
The parameter estimation of signals is one of important topics in electrical and electronics engineering area. For the estimation of main/fundamental parameters (amplitude, frequency and phase) of signals, many methods and techniques proposed. In this study, educational simulator for frequency estimation of signals is realized with artificial neural networks. Artificial neural networks are used for training Prony coefficients. Designed simulator is written in MATLAB and effects of neural net parameters (cost function, activation function, neuron size, etc.) on learning ability can be compared. Besides educational purposes, developed simulator can be used by engineers in order to create frequency estimators in practical studies.
\end{abstract}

Key words: Frequency estimation, Prony method, neural networks

\section{Introduction}

Signal parameter estimation is an important topic in the power electronics field. Especially, frequency estimation has crucial role in detecting signal quality. The frequency spectrum or harmonic spectrum of signals contains information of its total harmonic distortion [1]. For this reason, analysis of harmonic spectrum of signals is remarkable topic since early stages in power electronics [2-3]. Classical techniques of revealing harmonic content in signals are capable of only uncovering integer multiples of main harmonic [4-6]. If signal contains an inter-harmonic which is non-integer multiple of main harmonic, it couldn't be detected properly. In early stages of power electronics, there are no inter-harmonic sources in grids. But, after invention and usage of semiconductor devices, inter-harmonic concept is introduced in terminology. Therefore, many techniques are proposed recent years for detecting inter-harmonics [7-11]. One of important works for revealing inter-harmonic spectrum of signals dates back to XIX. century [12]. In this work, valuable signal parameter extraction technique was derived by Prony. This technique produces polynomial coefficients which are related with amplitude, phase and frequency information of signal. Based on these techniques, many hybrid methods are proposed in literature. Adaptive Linear Neuron (ADALINE) method is one of them [8]. Prony and ADALINE methods are discussed in section 2 in this paper.

In this study, Prony coefficients are tried to be estimated with ADALINE and non-linear artificial neural networks (ANN). Aim of this work, designing a simulator, which gives opportunity to student designing different ANN topologies in order to understand and comprehend how ANN could be used in frequency estimation. Signal model is derived from Photovoltaic systems (PV) which are run with Perturb and Observe Maximum Power Point Algorithm (P\&O MPPT) [13].

\section{Materials and Method}

\subsection{Data model}

*Corresponding author: Address: Faculty of Engineering, Department of Electrical-Electronics Engineering, Bursa Uludağ University, 16059, Bursa TURKEY. E-mail address: fahriv@uludag.edu.tr, Phone: +902242940905 
Data model which is used in this study is based on P\&O MPPT algorithm [13]. Proposed mathematical model is implemented in simulator which is created in MATLAB [14] and test data are produced. Mathematical expression is showed in Eq. (1). In this equation, $i_{g}$ is generated current by MPPT algorithm, $f_{g}$ is fundamental frequency, $f_{n}$ is harmonic frequency which is affected by MPPT frequency, $A_{n}$ and $\emptyset_{n}$ amplitude and phase values which are created by step voltage.

$$
\left.\begin{array}{c}
i_{g}(t)=\sum_{n=1}^{\infty} \frac{A_{n}}{2}\left[\cos \left(2 \pi t\left(f_{g}-f_{n}\right)+\emptyset_{n}\right)-\cos \left(2 \pi t\left(f_{g}+f_{n}\right)+\emptyset_{n}\right)\right] \\
a_{n}=\frac{2 V_{\text {step }}}{\pi n} \sin \left(\frac{\pi n}{2}\right), b_{n}=\frac{2 V_{\text {step }}}{\pi n} \cos (\pi n-1) \\
A_{n}=\sqrt{a_{n}^{2}+b_{n}^{2}}, \emptyset_{n}=\tan ^{-1}\left(\frac{b_{n}}{a_{n}}\right) \\
f_{n}=\frac{(2 n-1) f_{M P P T}}{4}
\end{array}\right\}
$$

\subsection{Prony method}

Fourier analysis can be extended as Eq. (2). In this equation, damping factor ' $\sigma$ ' is added to classical Fourier analysis. Solving technique of this eqsuation is called as Prony Method with respected to his work. Steps in Prony method can be divided in three steps [15-18].

$$
\hat{y}(t)=\sum_{i=1}^{\infty} A_{i} e^{\sigma_{i} t} \operatorname{Cos}\left(2 \omega f_{i} t+\varphi_{i}\right)
$$

- Step 1: Linear prediction model (LPM) is constructed based on observed data.

$$
\begin{gathered}
y_{N}=a_{1} y_{N-1}+a_{1} y_{N-1}+\cdots+a_{M} y_{N-M} \\
\underbrace{\left[\begin{array}{c}
y_{M} \\
y_{M+1} \\
\vdots \\
y_{N-1}
\end{array}\right]}_{\boldsymbol{S}}=\underbrace{\left[\begin{array}{cccc}
y_{M-1} & y_{M-2} & \cdots & y_{0} \\
y_{M} & y_{M-1} & \cdots & y_{1} \\
\vdots & \vdots & \ddots & \vdots \\
y_{N-2} & y_{N-3} & \cdots & y_{N-M-1}
\end{array}\right]}_{\boldsymbol{R}} \underbrace{\left[\begin{array}{c}
a_{1} \\
a_{2} \\
\vdots \\
a_{M}
\end{array}\right]}_{\boldsymbol{a}}
\end{gathered}
$$

- Step 2: Calculate roots of characteristic polynomial (Prony polynomial) which are obtained from LPM.

$$
\mu^{M}-a_{1} \mu^{M-1}-\cdots-a_{M-1} \mu-a_{M}=0
$$

- Step 3: Find complex amplitude, phase and frequency values with using roots which are calculated from Step 2. 


$$
\left[\begin{array}{c}
y_{0} \\
y_{1} \\
\vdots \\
y_{N-1}
\end{array}\right]=\left[\begin{array}{cccc}
1 & 1 & \cdots & 1 \\
\mu_{1} & \mu_{2} & \cdots & \mu_{M} \\
\vdots & \vdots & \ddots & \vdots \\
\mu_{1}^{N-1} & \mu_{2}^{N-1} & \cdots & \mu_{M}^{N-1}
\end{array}\right]\left[\begin{array}{c}
d_{1} \\
d_{2} \\
\vdots \\
d_{M}
\end{array}\right]
$$

\subsection{Usage of ANN in frequency and complex amplitude estimation}

Solving technique of Eq. (2) is called as Prony Method with Adaptive Linear Neuron (ADALINE) algorithm is proposed for finding signal parameters [8, 16]. ADALINE algorithm firstly calculates Prony polynomial coefficients based on Eq. (3). Frequency information is obtained from Prony polynomial coefficients. Then, second ADALINE network, which is fed by found frequency, is constructed. Using second ADALINE, amplitude and phase information is obtained. This procedure is shown in Fig. 1 and Fig. 2 [8].

$$
\left.\begin{array}{c}
\hat{y}=\sum_{m=1}^{M} A_{m}^{*} \sin \left(2 \pi f_{m}^{*} k \Delta t+\phi_{m}^{*}\right) \\
\hat{y}=\sum_{m=1}^{M}\left(A_{m}^{*} \cos \phi_{m}^{*} \sin 2 \pi f_{m}^{*} k \Delta t+A_{m}^{*} \sin \phi_{m}^{*} \cos 2 \pi f_{m}^{*} k \Delta t\right) \\
\hat{y}=\sum_{m=1}^{M}\left(\omega_{2 m-1}^{*} \sin \theta_{m}^{*}+\omega_{2 m}^{*} \cos \theta_{m}^{*}\right) \\
\omega_{2 m-1}^{*}=A_{m}^{*} \cos \phi_{m}^{*} \\
\omega_{2 m}^{*}=A_{m}^{*} \sin \phi_{m}^{*} \\
\theta_{m}^{*}=2 \pi f_{m}^{*} k \Delta t \\
\mathbf{x}^{*}(k)=\left[\omega_{1}^{*} \omega_{2}^{*} \ldots \omega_{2 M-1}^{*} \omega_{2 M}^{*}\right] \\
\mathbf{x}^{*}(k)=\left[\sin \theta_{1}^{*} \cos \theta_{1}^{*} \ldots \sin \theta_{M}^{*} \cos \theta_{M}^{*}\right]
\end{array}\right\} \Rightarrow \hat{y}=\mathbf{w}^{*}(k) \mathbf{x}^{*}(k)
$$

In back-propagation network, calculation of Prony polynomial coefficients has a nuance. Since every input has relations with each neuron, sums of weights of neural nets should be considered. If it is assumed that one hidden layer exists, calculation of Prony polynomial coefficients is given in Equation (10). In Equation (10), $a_{k}$ is Prony polynomial coefficient, $k$ is coefficient number, $I_{k, p}$ is coefficient of neural net, which corresponds to kth coefficient of neural net and pth neuron, $p$ is neuron number.

$$
a_{k}=\sum_{k=1}^{n} I_{k, p}
$$

In this study, based on schematic which is depicted in Fig. 1 and Fig. 2, back-propagation neural network simulator is designed in MATLAB. In back-propagation, every input has relation with every neuron, thus, LPM matrix is calculated with contribution of more neurons than ADALINE. 


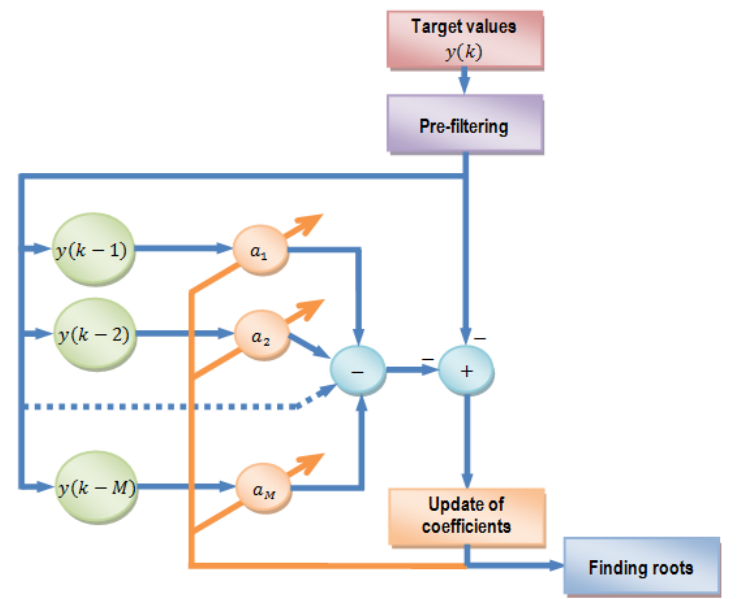

Figure 1. Frequency estimation state of ADALINE (Stage-1) [8]

\begin{tabular}{l}
\hline Start of Stage-1 \\
$\% \boldsymbol{P}$ is Prony polynomial, $\boldsymbol{Q 1}$ is ADALINE network, $\boldsymbol{a}$ is weights \\
of $Q 1$ and coefficient of $\boldsymbol{P}, \mathrm{n}$ is length of $\boldsymbol{a} \boldsymbol{S}$ is output of $Q 1, \boldsymbol{R}$ is \\
input of $Q 1, \mathbf{z}$ is roots of $\boldsymbol{P}, \Delta \boldsymbol{t}$ is sampling time \\
1. Specify $n$ \\
2. Train $\boldsymbol{Q 1}$ with outputs $\boldsymbol{P}$ and inputs $\boldsymbol{R}$ \\
3. Find $\boldsymbol{a}$ coefficients (weights) \\
4. If error is not acceptable go to 2 \\
5. Construct Prony polynomial with $\boldsymbol{a}$ \\
$6 . \quad$ Find $\mathbf{z}$ \\
7. Frequencies $=\operatorname{imag}(\ln (z) / 2 \pi \Delta t)$ \\
End of Stage-1
\end{tabular}

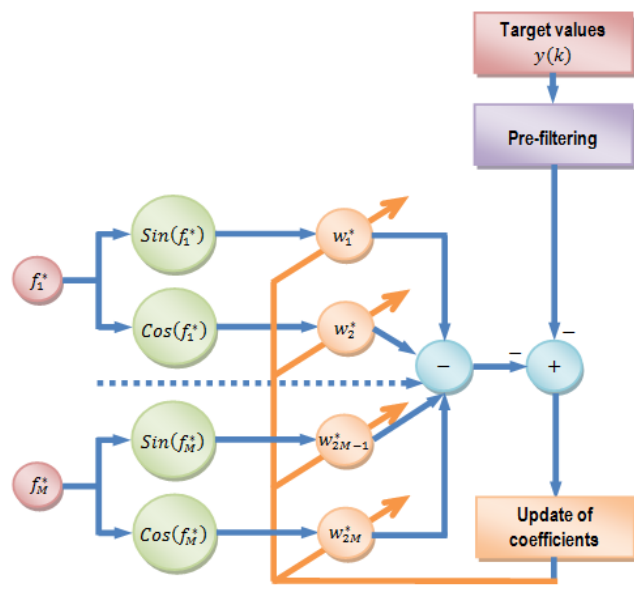

Figure 2. Amplitude and phase estimation state of ADALINE (Stage-2) [8]

Start of Stage-2
$\% \boldsymbol{Q 2}$ is ADALINE network, $\mathbf{w}^{*}(k)$ is coefficient vector of $Q 2$ network,
$w_{k}$ is element of $\mathbf{w}^{*}$ vector, $\boldsymbol{y}$ is analyzed signal and output of $Q 2$,
$\mathbf{x}^{*}(k)$ is input vector
1. Calculate $\mathbf{x}^{*}(k)$ vector based on found frequencies in first
2. Trep
3. If error is not acceptable go to 2
4. Phases $=$ atan $\left(w_{k} / w_{k+1}\right)(\mathrm{k}$ is odd value)
5. Amplitudes $=\sqrt{\left(w_{k}{ }^{2}+w_{k+1}\right)}$ (k is odd value)
End of Stage-2

\section{Simulations}

In this section, simulations are realized with ADALINE and back-propagation networks. The flowchart of designed simulator is given in Fig. 3. Designed simulator is run on MATLAB and Deep Learning Toolbox [14, 19]. In order to show an example, ADALINE and non-linear network (which is implemented in this simulator) can be compared. Sampling period of test signal which is derived from mathematical model of P\&O MPPT method is selected as $1 \mathrm{~s}$ time duration is specified as $0.6 \mathrm{~s}$. Step voltage of PV system is $12 \mathrm{~V}$ and MPPT frequency is $20 \mathrm{~Hz}$.

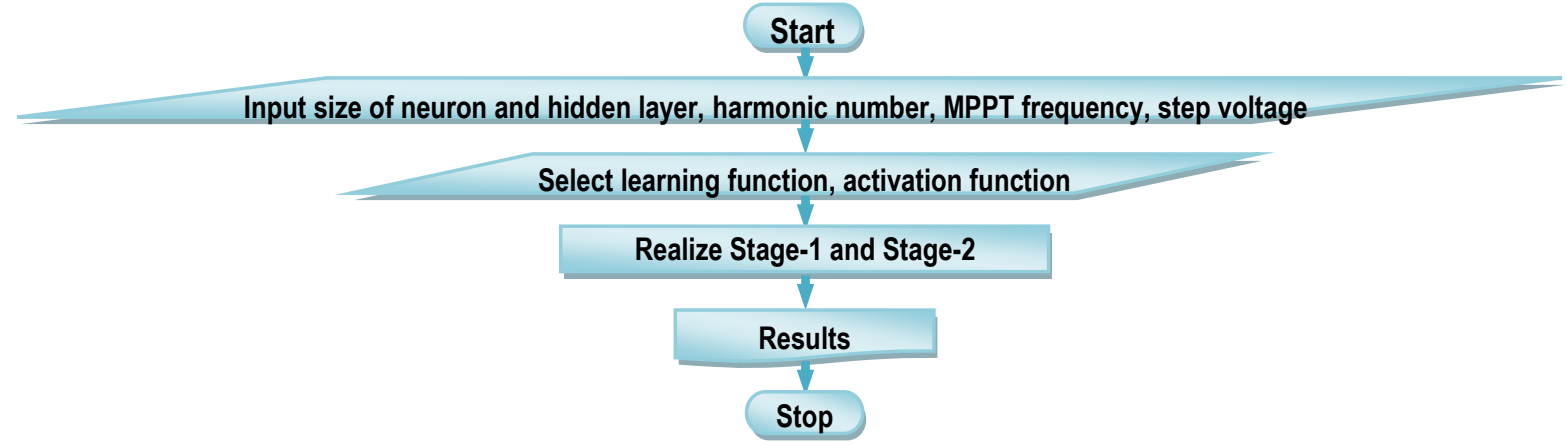

Figure 3. The flowchart of designed simulator 


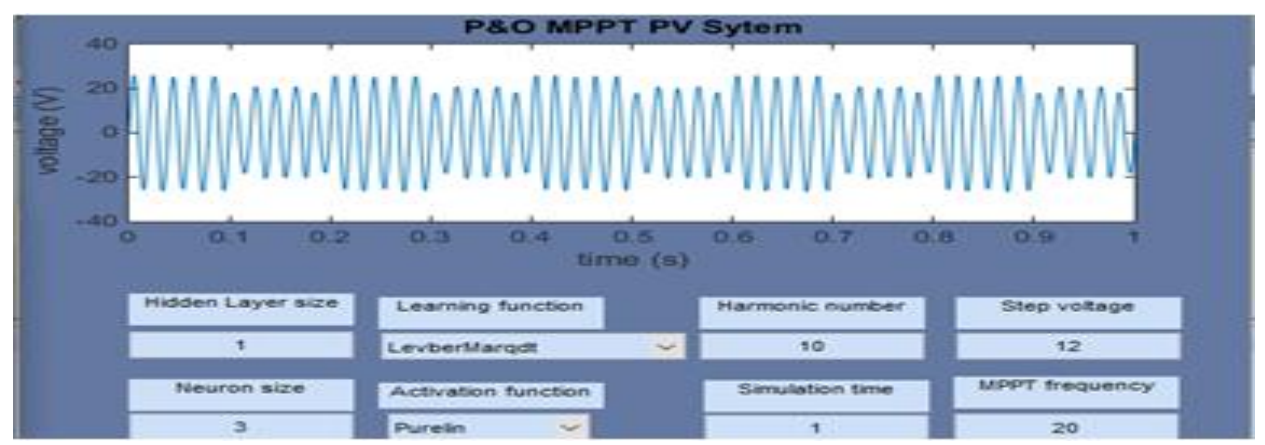

Figure 4. Simulation screen

In ADALINE simulation, 750 inputs are used. In back-propagation, 400 inputs are used. Backpropagation model consists of one input layer, one output layer and one hidden layer with 3 neurons. In complex amplitude calculation, two times of found frequency components should be fed to input of second neural network as stated in Equation (9). Therefore, 400 hundred inputs are changed to 800 hunders inputs for second network which uncovers complex amplitude values. Obtained results are shown in Table 1. Simulation screens for ADALINE and different form of back-propagation algorithm is shown in Fig. 4.

Table 1. Obtained results with ADALINE and back-propagation for frequency estimation $\left(\boldsymbol{V}_{\text {step }}=\mathbf{1 2 V}\right.$ and $\left.\boldsymbol{f}_{M P P T}=\mathbf{5 H z}\right)$

\begin{tabular}{|c|c|c|c|c|}
\hline Real/True value & \multicolumn{2}{|c|}{ ADALINE } & \multicolumn{2}{c|}{ Back-propagation } \\
\hline Frequency (Hz) & Estimation (Hz) & Relative error (\%) & Estimation (Hz) & Relative error (\%) \\
\hline 5 & 4.8989 & 2.0220 & 5.0000 & $\sim 0$ \\
\hline 15 & 17.3109 & 15.4060 & 15.0000 & $\sim 0$ \\
\hline 25 & 23.1178 & 7.5288 & 25.0000 & $\sim 0$ \\
\hline 35 & 35.4164 & 1.1897 & 35.0000 & $\sim 0$ \\
\hline 45 & 44.0699 & 2.0669 & 45.0000 & $\sim 0$ \\
\hline 50 & 49.4533 & 1.0934 & 50.0000 & $\sim 0$ \\
\hline 55 & 55.1027 & 0.1867 & 55.0000 & $\sim 0$ \\
\hline 65 & 65.3665 & 0.5638 & 65.0000 & $\sim 0$ \\
\hline 75 & 76.8383 & 2.4511 & 75.0000 & $\sim 0$ \\
\hline 85 & 86.4204 & 1.6711 & 95.0000 & $\sim 0$ \\
\hline 95 & 97.8306 & 2.9796 & 95.0000 & \\
\hline
\end{tabular}

It is important to note that this simulator gives opportunity to its users how new neural topology could be designed with differentiating existing one. ADALINE is a linear network, and every input has one weight. But, in back-propagation, 3 neurons are used and every input has weights to each neuron (400x3 weights). In back-propagation, sum of weight which is directly connected to specific input should be considered as Prony polynomial coefficients and this procedure is given in Equation (10).

\section{Conclusions}

In this work, an educational simulator is designed for frequency estimation, using ANNs. It is designed in MATLAB and Deep Learning Toolbox. In this simulator, various ANN types with different learning function, neuron size, layer size, etc. can be designed and investigated. It gives opportunity to its users to analyze existing topologies and creating new schemes in order to adapt new problems. 


\section{References}

[1] Associated Power Technologies. Total harmonic distortion and effects in electrical power systems. 2011.

[2] Bedell F, Mayer EC. Distortion of alternating-current wave caused by cyclic variation in resistance. Transactions of the American Institute of Electrical Engineers 1915; XXXIV (1): 333-348. doi: https://doi.org/10.1109/T-AIEE.1915.4765220

[3] Bedell F, Tuttle EB. The effect of iron in distorting alternating-current wave form. Proceedings of the American Institute of Electrical Engineers 1906; 25(9): 601-621. doi: https://doi.org/10.1109/PAIEE.1906.6742509

[4] Testa A et al.. Interharmonics: Theory and modeling. IEEE Transactions on Power Delivery 2007; 22(4): 2335-2348. doi: https://doi.org/10.1109/TPWRD.2007.905505

[5] Duhamel P, Vetterli M. Fast Fourier transforms: A tutorial review and a state of the art. Signal Processing 1990; 19(4): 259-299. doi: https://doi.org/10.1016/0165-1684(90)90158-U

[6] Cooley JW, Tukey JW. An algorithm for the machine calculation of complex Fourier series. Mathematics of Computation 1965; 19(90): 297-301. doi: https://doi.org/10.1090/S0025-5718-1965-0178586-1

[7] Xiong J, Wang B, Zhang S. Interharmonics analysis based on windowed interpolation and prony algorithm. 2010 2nd International Asia Conference on Informatics in Control, Automation and Robotics (CAR 2010), Wuhan, 2010, pp. 430-433, doi: https://doi.org/10.1109/CAR.2010.5456806

[8] Chang GW, Chen C-I, Liang Q-W. A two-stage ADALINE for harmonics and interharmonics measurement. IEEE Transactions on Industrial Electronics 2009; 56(6): 2220-2228. doi: https://doi.org/10.1109/TIE.2009.2017093

[9] Subudhi B, Ray PK. Estimation of power system harmonics using hybrid RLS-Adaline and KF-Adaline algorithms. TENCON 2009 - 2009 IEEE Region 10 Conference, Singapore, 2009, pp. 1-6, doi: https://doi.org/10.1109/TENCON.2009.5396102

[10] Chen H, Sun Y, Cheng Y. Harmonic and inter-harmonic detection of grid-connected distributed generation based on modified mathematical morphology filter and Hilbert-Huang transformation. 2009 IEEE 6th International Power Electronics and Motion Control Conference, Wuhan, 2009, pp. 1155-1160. doi: https://doi.org/10.1109/IPEMC.2009.5157557

[11] Schmidt R. Multiple emitter location and signal parameter estimation. IEEE Transactions on Antennas and Propagation 1986; 34(3): 276-280. doi: https://doi.org/10.1109/TAP.1986.1143830

[12] Prony R. Essai expérimental et analytique: sur les lois de la dilatabilité de fluides élastique et sur celles de la force expansive de la vapeur de l'alcool, à différentes températures. Journal de l'École Polytechnique Floréal et Plairial 1795; 1(22): 24-76.

[13] Sangwongwanich A, Yang Y, Sera D, Soltani H, Blaabjerg F. Analysis and modeling of interharmonics from grid-connected photovoltaic systems. IEEE Transactions on Power Electronics 2018; 33(10): 8353-8364. doi: https://doi.org/10.1109/TPEL.2017.2778025

[14] MathWorks. MATLAB \& Simulink. 2018.

[15] Hauer JF, Demeure CJ, Scharf LL. Initial results in Prony analysis of power system response signals. IEEE Transactions on Power Systems 1990; 5(1): 80-89. doi: https://doi.org/10.1109/59.49090

[16] Yalcin NA, Vatansever F. Comparison of Prony and ADALINE method in inter-harmonic estimation. Uludağ University Journal of The Faculty of Engineering 2020; 25(1): 405-418. doi: https://doi.org/10.17482/uumfd.592988

[17] Vatansever F, Cengelci B. Prony yöntemiyle harmonik analizi. 6th International Advanced Technologies Symposium (IATS'11), Turkey, 2011, pp. 134-137.

[18] Chang GW, Chen C. Measurement techniques for stationary and time-varying harmonics. IEEE PES General Meeting, Providence, RI, 2010, pp. 1-5. doi: https://doi.org/10.1109/PES.2010.5589611

[19] MathWorks. MATLAB \& Deep Learning Toolbox. 2018. 